\title{
Comparing different contour methods with response-based methods for extreme ship response analysis
}

\author{
Erik Vanem*, Bingjie Guo \\ DNV GL Group Technology and Research, Høvik, Norway \\ Emma Ross \\ Shell Global Solutions, Amsterdam, The Netherlands \\ Philip Jonathan \\ Shell Research Ltd., London, United Kingdom \\ Lancaster University, Department of Mathematics and Statistics, Lancaster, United Kingdom
}

\begin{abstract}
Environmental contours are often applied in probabilistic structural reliability analysis to identify extreme environmental conditions that may give rise to extreme loads and responses. They facilitate approximate long term analysis of critical structural responses in situations where computationally heavy and time-consuming response calculations makes full long-term analysis infeasible. The environmental contour method identifies extreme environmental conditions that are expected to give rise to extreme structural response of marine structures. The extreme responses can then be estimated by performing response calculations for environmental conditions along the contours.

Response-based analysis is an alternative, where extreme value analysis is performed on the actual response rather than on the environmental conditions. For complex structures, this is often not practical due to computationally heavy response calculations. However, by establishing statistical emulators of the response, using machine learning techniques, one may obtain long time-series of the structural response and use this to estimate extreme responses.

In this paper, various contour methods will be compared to response-based estimation of extreme vertical bending moment for a tanker. A response emulator based on Gaussian processes regression with adaptive sampling has been established based on response calculations from a hydrodynamic model. Long timeseries of sea-state parameters such as significant wave height and wave period are used to construct Nyear environmental contours and the extreme $\mathrm{N}$-year response is estimated from numerical calculations for identified sea states. At the same time, the response emulator is applied on the time series to provide long time-series of structural response, in this case vertical bending moment of a tanker. Extreme value analysis is then performed directly on the responses to estimate the N-year extreme response. The results from either method will then be compared, and it is possible to evaluate the accuracy of the environmental contour method in estimating the response. Moreover, different contour methods will be compared.
\end{abstract}

Keywords: Extreme ship response analysis, marine structures, Environmental contours, Structural reliability, Response-based methods, ocean environment, environmental loads

\section{Introduction and Background}

Environmental contours are often used in long-term extreme response analysis of marine structures as a simple and approximate alternative to more computationally demanding full long-term analyses. One of

\footnotetext{
${ }^{*}$ Corresponding author

Email address: Erik.Vanem@dnvgl.com (Bingjie Guo)
} 
the main advantages of environmental contours is that the structural response analysis is de-coupled from

5 the environmental description, meaning that only a limited number of short term response calculations are needed for long-term analysis. That is, the environmental contours method identifies critical environmental conditions for which short term analysis is performed [1. Response-based analysis is an alternative to contour-based methods, if knowledge about the response is known or if a reasonable approximator for the response can be established at affordable computational cost.

Environmental contours were first proposed as equi-density lines [2, but currently contours based on the inverse first order reliability method (IFORM) are more widely used [3, 4]. More recently, environmental contours based on direct Monte Carlo sampling have been proposed [5, 6]. These contours have well-defined probabilistic properties, and will in some cases be quite different from the traditional IFORM-contours, see the comparison studies in [7, 8] and also [9. In particular, the direct sampling contours will by definition

15 always enclose a convex set, whereas the IFORM contours will tend to follow the scatter of the data more closely. Other approached for environmental contours exist, see e.g. [10, 11, 12, 13, 14, 15, 16, 17] or the recent review in [18].

In this paper various approaches to environmental contours will be used, and the results from an approximate long-term analysis using such contours will be compared to an approximate long-term analysis based on a statistical response emulator. Moreover, different modelling approaches for the long-term wave description will be explored, including an initial distribution approach and a conditional extremes modelling approach. A recent comparison study of response-based and contour-based methods was presented in [19]. Moreover, parts of the study presented herein was presented in [20. In the following, a brief introduction to the different methods for constructing environmental contours will be given, as well as an introduction to

25 Gaussian processes models which has been used for the response emulator.

\subsection{Environmental contours}

The direct sampling approach to environmental contours utilizes Monte Carlo sampling from a joint distribution of environmental parameters in order to draw contour lines corresponding to a target exceedance probability. That is, each segment of the environmental contour correspond to a hyperplane with a specified so exceedance probability, and under the assumption of a convex failure region this will represent an upper bound on the failure probability of a structure. The full recipe for calculating environmental contours with the direct sampling approach will not be repeated herein, but reference is made to previous publications where this is outlined in detail [5, 6, 21. In short, the environmental contours are calculated as follows (in the 2-dimensional case, higher dimensional contours can be constructed in a similar way, see e.g. [22]): Let

${ }_{35} P_{e}$ be the target exceedance probability. The first step is to simulate a sufficiently large number of Monte Carlo samples from the joint distribution model. Then for any given angle $\theta \in[0,360)$, identify a straight line $\Pi(\theta)$ defined by an equation on the form $t \cos (\theta)+h \sin (\theta)=C(\theta)$, partitioning the sample space into two halfspaces $\Pi(\theta)^{+}$and $\Pi(\theta)^{-}$such that the fraction of sample points in $\Pi(\theta)^{+}$is approximately equal to $P_{e}$. The resulting set $\mathcal{B}$ is then obtained by intersecting the sets $\Pi(\theta)^{-}$for all $\theta \in[0,360)$, and constitute the environmental contours.

The IFORM approach to environmental contours is outlined in [3, 4], and essentially finds environmental contour by transforming a circle with radius corresponding to the target reliability level in standard normal space into the space of the environmental variables, in this case significant wave height and wave period. The resulting contours will describe the extreme wave climate based on exceedances probabilities in the standard

45 normal space and is based on a linear approximation of the limit state function in that space. One difference between the direct sampling contours and the IFORM-based contours is that the direct sampling contours will always be convex, whereas the IFORM contours need not be, see also [7, 8].

Two other contour methods are applied in this study for the conditional extremes model. That is, joint exceedance contours and isodensity contours. Joint exceedance contours are based on a definition proposed

so in [23. In this study, isodensity contours are estimated from simulation under a conditional extremes model; further description is given in Section 2.2 .

The calculation of environmental contours is based on a joint distribution describing the relevant metocean parameters, for example the significant wave-height $H_{s}$ and peak wave-period $T_{p}$. The joint distribution is in turn normally based on some dataset. There are uncertainties related to the fitting of a joint distribution to

55 data and the results strongly depend on the choice of parametric model 24] and other choices made during the fitting procedure 25]. Moreover, there is also an uncertainty related to the fact that the underlying metocean 
data is of finite sample size [26]. These uncertainties will obviously be propagated to the environmental contours, and should be acknowledged when applying contour methods [27, 28].

\subsection{Gaussian processes}

6о A stochastic process is a collection of random variables typically associated with a set of continuous indices such as space, time or any other input variables. A Gaussian process is a stochastic process where every realization of the process, that is, every finite collection of the random variables, have a multivariate normal (Gaussian) distribution, and it can be regarded as an infinite-dimensional generalization of the multivariate normal distribution.

The distribution of a set of multivariate normal random variables is fully specified by its mean vector $\boldsymbol{\mu}$, specifying the expected value of each random variable, and its covariance matrix $\boldsymbol{\Sigma}$, specifying the variances of each random variable as well as the covariance between any pair of random variables. The density of a collection of random variables $\boldsymbol{X}^{T}=\left(X_{1}, X_{2}, \ldots, X_{k}\right)$ which are multivariate normally distributed with mean vector $\boldsymbol{\mu}$ and covariance matrix $\boldsymbol{\Sigma}$ is

$$
f(\boldsymbol{x})=\frac{1}{\sqrt{(2 \pi)^{k}|\boldsymbol{\Sigma}|}} e^{-\frac{1}{2}(\boldsymbol{x}-\boldsymbol{\mu})^{T} \boldsymbol{\Sigma}^{-1}(\boldsymbol{x}-\boldsymbol{\mu})} .
$$

65

One important feature of the multivariate normal distribution is that if a set of random variables are jointly multivariate normally distributed, then the marginal distribution of any of the random variables will be Gaussian. Moreover, the conditional distribution of any of the random variables given the others will also be normal. In Gaussian processes, the mean vector is replaced by the mean function and the covariance matrix is replaced by a covariance function, which are typically continuous functions determining the process' behaviour over the input space. Having specified these functions, the Gaussian process is fully specified over the input space. For a homogeneous Gaussian process, the covariance function, $\kappa\left(\boldsymbol{x}, \boldsymbol{x}^{\prime}\right)$ will only depend on the distance between points in the input space, $d=\left|\boldsymbol{x}-\boldsymbol{x}^{\prime}\right|$.

In Gaussian processes regression, the properties of Gaussian processes are exploited, and if a random process can be modelled as a Gaussian process, it may be used to predict the distribution at unobserved 75 points in the input space. Such predictions will not only be point predictions, but it will provide the marginal distributions at unobserved points (which will be a marginal normal distribution) and hence also provide an estimate of the uncertainty. Given a set of observations from the Gaussian process, predictions may be the conditional distributions given the observed values. Typically, in Gaussian processes regression, the dependent variables $\boldsymbol{Y}$ are modelled as a Gaussian process over the space of the input variables, $\boldsymbol{X}$, and

so the training data set is used to estimate the mean- and covariance functions as well as to provide predictive distributions for unobserved points in input space.

In order to train a Gaussian process model, there is a need to specify the type of covariance function and in this study the radial basis kernel function is used. This kernel function has one hyper parameter, the inverse kernel width, $\sigma$, and takes the following form:

$$
\kappa\left(\boldsymbol{x}, \boldsymbol{x}^{\prime}\right)=e^{-\frac{1}{2 \sigma^{2}}\left\|\boldsymbol{x}-\boldsymbol{x}^{\prime}\right\|^{2}} .
$$

This kernel is also sometimes referred to as the Gaussian or the squared exponential kernel. In addition, a nugget effect or variance may be included to account for noisy data, and this may typically be a constant value, for example on the form $c \delta(i, j)$ where $\delta(\cdot)$ denote the delta function and $c$ is the noise variance.

85 Estimation can be done by maximum likelihood or by Bayesian methods [29].

\section{Wave data description}

A good wave climate description is needed in order to calculate ship responses in realistic sea states. In this study, non-linear numerical wave models have been used to simulate ocean waves given concurrent values of the sea state parameters significant wave height and spectral peak wave period, $\left(H_{S}, T_{P}\right)$. Hence,

9o a probabilistic description of the wave climate in terms of these variables are needed and the input to both numerical response calculations and construction of environmental contours are joint data for $H_{S}$ and $T_{P}$. For the purpose of this study, wave climate data from numerical model runs have been used for one location in the North Atlantic Ocean. The data consist of 3 sets of 30-year time-series for the period January 1971 
- December 2000 corresponding to three ensemble members from model runs, as described in [30, 31]. The 95 location of the data is $57.62^{\circ} \mathrm{N}, 20.28^{\circ} \mathrm{W}$. It is assumed that the three model runs are three independent and equally likely realizations of the ocean wave climate at this location and these data will be utilized in this study. Hence, results will be presented for each of the subsets of 30-year data, as well as a combined dataset from all three ensembles. In the latter case, it is tacitly assumed that the combined data represents 90 years of wave climate data for a stationary wave climate. Scatterplots of the three datasets are presented in Figure 1.

\section{Scatterplot of the wave data}

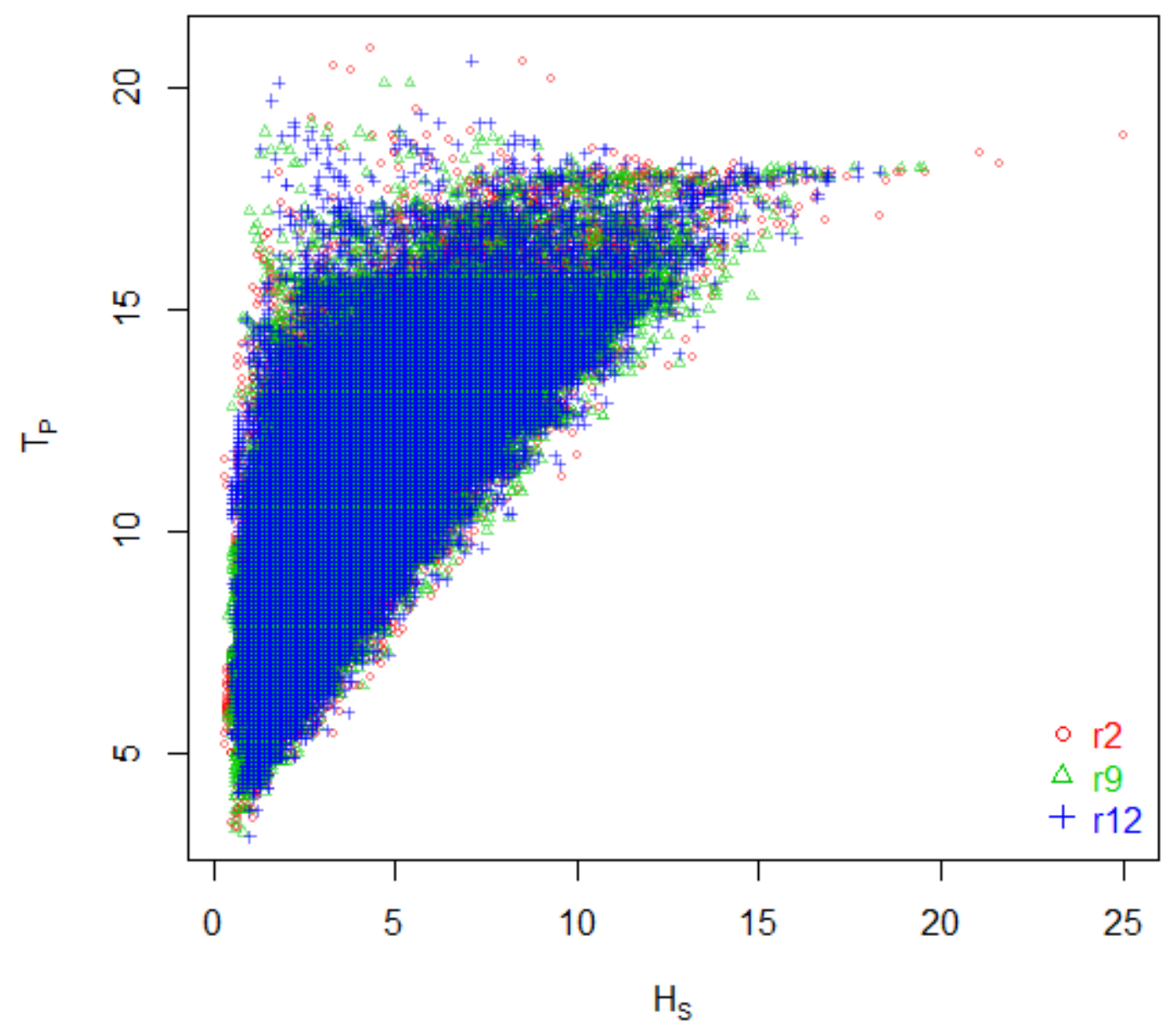

Figure 1: Scatterplots of the wave climate data.

In this paper, two different approaches will be used to describe the underlying distribution of the wave climate based on the available data. The first uses all the data and fits a parametric joint distribution function to the data. The second approach uses peak-picking and fits a conditional extremes model to the jointly extreme data obtained in the peak-picking step. These models will be further outlined in the following.

\subsection{A Weibull-log-normal model for sea state $H_{s}, T_{P}$, and estimated environmental contours}

The datasets described in the previous section have been used to fit parametric distribution models for $H_{S}$ and $T_{P}$ in order to establish environmental contours for probabilistic reliability assessment. There are 
Table 1: Distribution parameters estimated to the various datasets for the sea states.

\begin{tabular}{lccccccccc}
\hline & \multicolumn{3}{c}{3 -parameter Weibull $\left(H_{S}\right)$} & \multicolumn{4}{c}{ Conditional log-normal $\left(T_{P}\right)$} \\
& (scale) & (shape) & (location) & \multicolumn{3}{c}{ (log-mean) } & \multicolumn{3}{c}{ (log-std) } \\
& $\alpha$ & $\beta$ & $\gamma$ & $a_{1}$ & $a_{2}$ & $a_{3}$ & $b_{1}$ & $b_{2}$ & $b_{3}$ \\
\hline r2 & 2.426 & 1.195 & 1.327 & 0.678 & 1.378 & 0.165 & 0.0150 & 0.193 & -0.122 \\
r9 & 2.630 & 1.253 & 1.120 & 1.565 & 0.513 & 0.339 & 0.0150 & 0.200 & -0.129 \\
r12 & 2.727 & 1.288 & 0.1 .083 & 1.854 & 0.266 & 0.492 & 0.0150 & 0.0 .228 & -0.152 \\
Combined data & 2.609 & 1.251 & 1.165 & 1.203 & 0.871 & 0.231 & 0.0150 & 0.212 & -0.139 \\
\hline
\end{tabular}

many alternative ways of fitting a parametric model to such data [24], and for this study a conditional model with a marginal 3-parameter Weibull distribution for $H_{S}$ and a conditional log-normal distribution for $T_{P}$ is assumed, see also [32, as follows:

$$
\begin{gathered}
f_{H_{s}}\left(h_{s}\right)=\frac{\beta}{\alpha}\left(\frac{h_{s}-\gamma}{\alpha}\right)^{\beta-1} \exp \left[-\left(\frac{h_{s}-\gamma}{\alpha}\right)^{\beta}\right], \\
f_{T_{p} \mid H_{s}}\left(t_{p} \mid h_{s}\right)=\frac{1}{\sigma\left(h_{s}\right) t_{p} \sqrt{2 \pi}} \exp -\frac{\left(\ln t_{p}-\mu\left(h_{s}\right)\right)^{2}}{2 \sigma\left(h_{s}\right)^{2}},
\end{gathered}
$$

where

$$
\mu=E\left(\ln T_{p}\right)=a_{1}+a_{2} h_{s}^{a_{3}}, \quad \sigma=s t d\left(\ln T_{p}\right)=b_{1}+b_{2} e^{b_{3} h_{s}} .
$$

The model parameters for the marginal distribution have been fitted by minimizing the $2^{\text {nd }}$ order Anderson Darling statistic, and the parameters of the conditional model have been fitted by calculating conditional mean and standard deviation for binned data and fit the functions to these binned values by least square 110 fitting, as outlined in [8], see also [25].

The estimated model parameters are presented in Table 1, and the corresponding environmental contours for the 25-year conditions are shown in Figure 2. The differences between the contours can be ascribed to sampling variability [26] and all contours are assumed to represent an equally likely description of the 25-year extreme wave climate for the location under investigation. It is also observed that the contours based on the direct sampling approach and the IFORM describe similar extreme wave conditions, but that there are some differences with respect to the shape of the contours. As expected, the direct sampling contours are convex and the IFORM contours are not.

\subsection{A conditional extremes model for storm peak $H_{S}, T_{P}$, and associated environmental contours}

Joint models and contours in this section are based on a sample of storm peak events of $H_{S}$ and associated values of $T_{P}$. This sample is isolated from sea-state time-series using the procedure described by [33]. The conditional extremes model of [34], and its numerous extensions, provide a framework to estimate joint densities of $H_{S}$ and $T_{P}$, and hence design contours. The approach is motivated by the asymptotic form

$$
Y_{2} \mid\left\{Y_{1}=y\right\}=\alpha y+y^{\beta}(\mu+\sigma Z)
$$

for the limiting conditional behaviour of one variable ( $Y_{2}$ for definiteness) given a large value $y$ of a condi120 tioning variable $\left(Y_{1}\right)$. Parameters $\alpha \in[-1,1], \beta \in(-\infty, 1], \mu \in(-\infty, \infty)$ and $\sigma \in(0, \infty)$ are estimated from the data, together with a sample to represent the distribution of residual process $Z$. See [35] for details. To use the conditional extremes model, variables $Y_{1}$ and $Y_{2}$ must be expressed on standard Laplace marginal scales. This is achieved by first fitting marginal gamma-generalised Pareto models for each of $H_{S}$ and $T_{P}$ as described in [36], followed by transformation (to $Y_{1}, Y_{2}$ respectively) using the probability integral transform.

125 Simulation under the fitted conditional extremes model allow estimation of isodensity, joint exceedance and direct-sampling contours. 

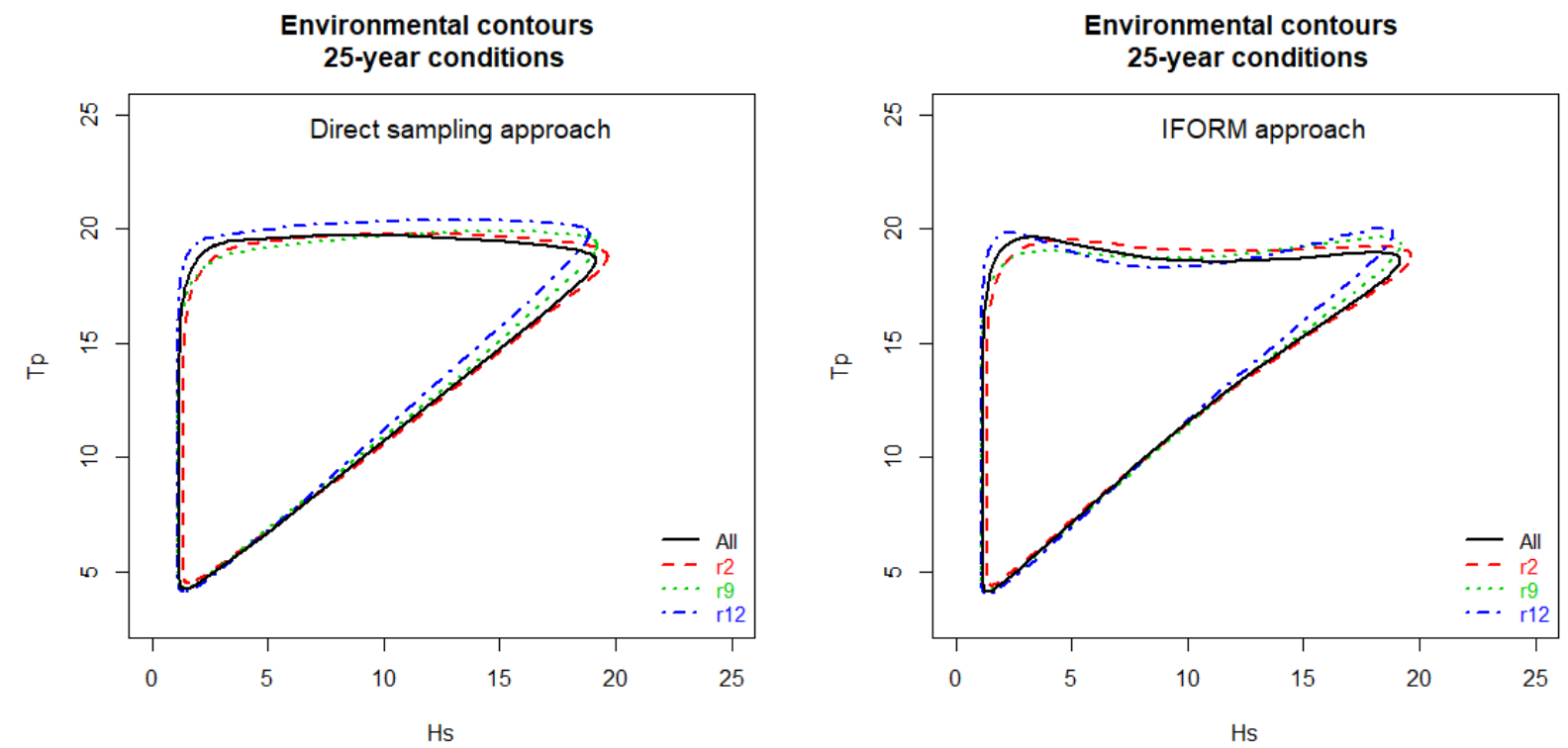

Figure 2: Estimated environmental contours for the different datasets; Direct sampling approach (left) and IFORM approach (right)

Table 2: Main parameters of the LNG tanker.

\begin{tabular}{lccc}
\hline & Unit & Full scale & Model scale \\
\hline Scale & {$[-]$} & 1 & $1 / 70$ \\
Length (Lpp) & {$[\mathrm{m}]$} & 186.90 & 2.670 \\
Breadth (B) & {$[\mathrm{m}]$} & 30.38 & 0.434 \\
Depth (D) & {$[\mathrm{m}]$} & 18.20 & 0.268 \\
Draught (T) & {$[\mathrm{m}]$} & 8.40 & 0.120 \\
Displacement $(\Delta)$ & {$[\mathrm{kg}]$} & 35674800 & 103.831 \\
$C O G_{x}$ & {$[\mathrm{~m}]$} & 94.8675 & 1.35525 \\
$C O G_{y}$ & {$[\mathrm{~m}]$} & 0 & 0 \\
$C O G_{z}$ & {$[\mathrm{~m}]$} & 8.26 & 0.118 \\
\hline
\end{tabular}

The three different environmental contours are shown for the three datasets as well as the combined dataset in Figure 3, based on a stationary analysis. Omni-directional-seasonal contours from the non-stationary analysis on the combined dataset are shown in Figure 4, where different seasonal-directional bins have been used. Contours from this non-stationary analysis for different directional-seasonal bins are shown in Figure 5

\section{Case study: Extreme vertical bending moment of an LNG tanker}

In order to make comparison of the environmental contour method and response-based analysis for extreme ship bending moment, one statistical emulator of the response for one LNG tanker is established using machine learning techniques, based on numerical simulation performed with Wasim combined with the nonlinear wave model UOSM [37, 38]. The numerical simulations and its verification are given first. Then the response emulator is discussed in the subsequent sub-section.

One LNG tanker has been chosen for this study. The main parameters of the tanker are given in Table 2 Model tests on the LNG tanker were performed in the sea-keeping basin of Technical University Berlin

140 (TUB) at model scale 1:70 within the EC EXTREME SEAS project. A more detailed description of the model test can be found in [39]. 

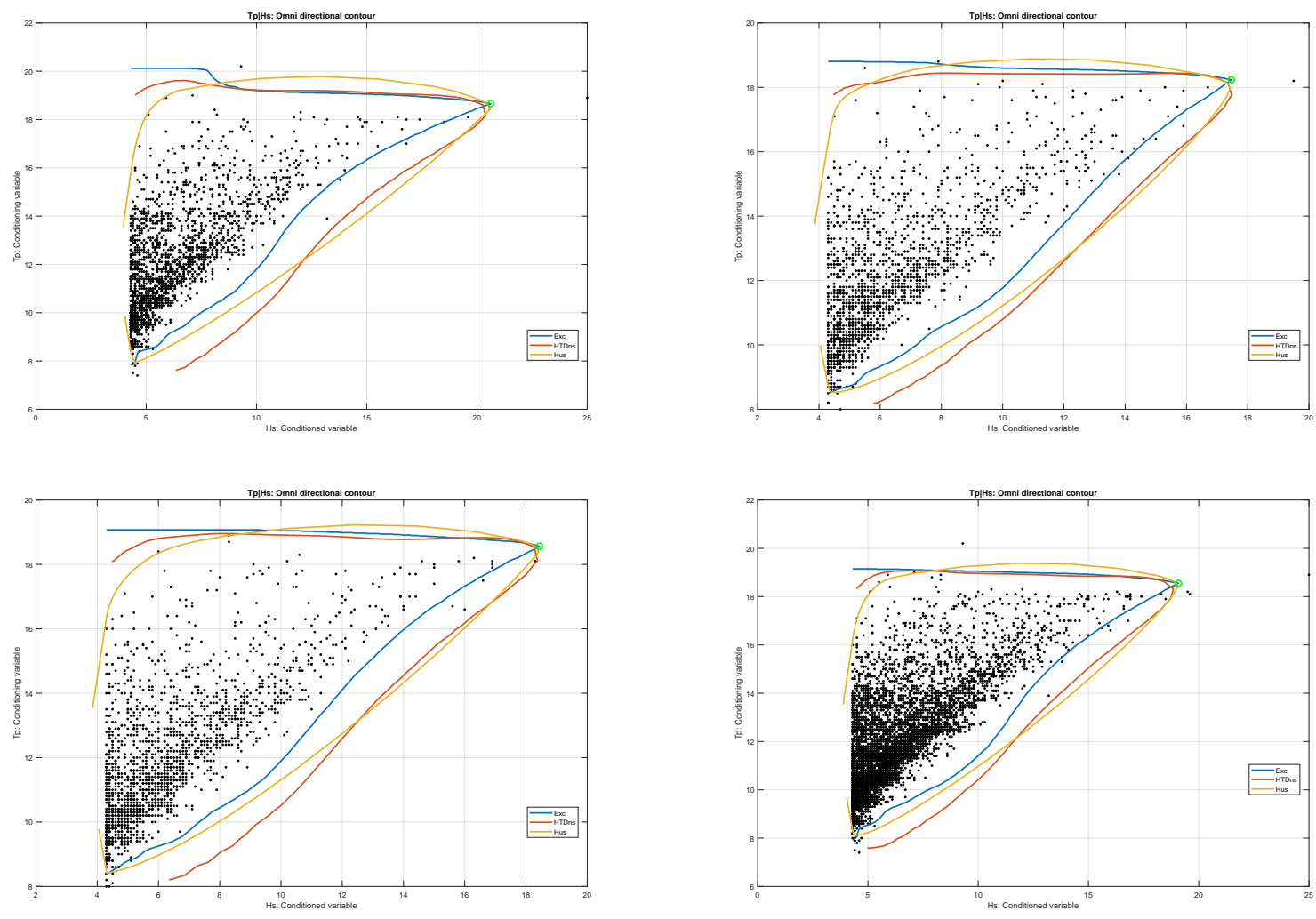

Figure 3: Estimated environmental contours for the different datasets with the conditional extremes model; R2 data (top left), R9 data (top right), R12 data (bottom left) and the combined data (bottom right)

\subsection{Response calculations and verification}

The 3-hours extreme bending moment is calculated with Wasim, with waves from nonlinear HOSM wave model. WASIM solves the 3D radiation/diffraction problem using the Rankine panel method. The nonlinear Froude-Krylov force and hydrostatic force can be added to account partly for nonlinear effects. The HOSM code used in this study is internally developed DNV GL HOSM, which implements the HOSM methods presented in [38. It also provides the full calculation of all water particle kinematics using the H2-operator method described in [40]. The HOSM code provides a consistent nonlinear calculation of the water particle kinematics to the given order $M$. In this study the nonlinear order was chosen as $M=5$, which includes the most important nonlinear effects, including bound waves up to fifth order.

The numerical simulations with the 3D panel method and HOSM wave model were verified with model test as reported in 41. The numerical simulation in linear waves were performed to verify the mesh and setting of the numerical model. The waves from the HOSM model and linear wave model are compared with model test to verify the HOSM wave model. The comparisons show that the HOSM model agrees better with model test than linear simulation. However, the HOSM model gives more extreme crests than experiments in some cases. The comparisons of ship bending moment of the LNG tanker from different numerical simulations with model tests show that nonlinear 3D panel method with the HOSM wave model slightly overestimates the ship hogging moment and gives the best prediction for the ship sagging moment. It was argued that the overestimation on wave crest by the HOSM model could be partly due to the fact that HOSM simulations

160 are run without a wave breaking model. In this study, HOSM with a breaking model is adopted for wave simulation.

The verification of HOSM with a breaking model is performed in one irregular wave with $T_{P}=12 s$, $H_{S}=9.7 \mathrm{~m}$ and a peak enhancement factor for the JONSWAP wave spectrum $\gamma=6$, as shown in Figure 6 The comparison is performed in full scale. Crest-HOSM and Trough-HOSM give the exceedance probability 


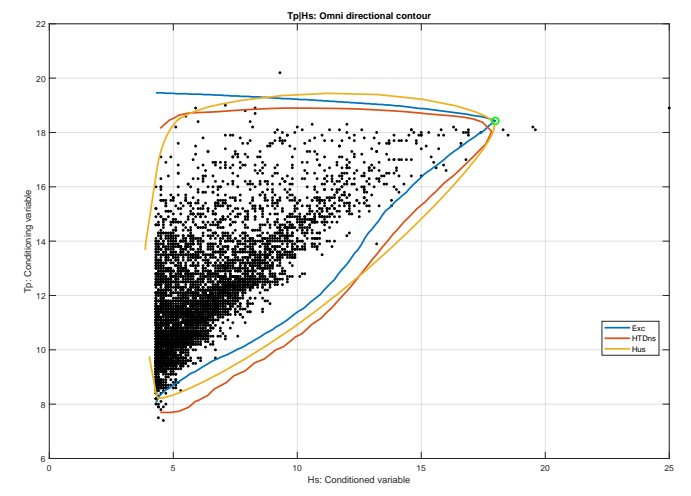

Figure 4: Omni-directional contours based on non-stationary analysis of the combined dataset

165 of wave crest and trough from the HOSM wave model without any breaking model. Crest-HOSM-BR and Trough-HOSM-BR are wave results from the HOSM wave model with breaking. Crest-LI and Trough-Li gives the results from linear wave model and Crest-exp and Trough-exp refers to results from the model tests. The comparison confirms that the HOSM wave model can give better wave estimation than a linear wave model, especially for wave crests. The inclusion of a breaking model can improve the numerical results in the upper tail of the distribution.

The different numerical calculations of ship bending moments in the selected irregular sea state are compared in Figure 7 to verify the numerical model adopted in this study. $x x-L I-L I$ are results from pure simulation, where both the 3D panel program and the wave model are linear. $x x-H O S M$ and $x x-H O S M-B R$ represent the results from partly nonlinear 3D panel program with the HOSM wave model without and with

175 a breaking model, respectively. $x x$-Exp denotes results from model tests. The results indicate that linear simulations tend to underestimate the ship sagging moment. The comparison also show that the breaking model can improve the accuracy, particularly for large bending moments. Thus, the partly nonlinear 3D panel program and the HOSM wave model with a breaking model are used to establish the response emulator outlined in the next section.

\subsection{Response emulator}

In order to perform response-based analysis, a method for efficiently approximating the response is needed and in this study a response emulator is established using Gaussian processes regression. 3-hours extreme bending moments are calculated for selected sea states, and these are used to train a Gaussian processes regression model, with bending moment as the target variable and $\left(T_{P}, H_{S}\right)$-values as explanatory variables.

185 In this study, the Gaussian processes regression model uses a constant kernel combined with a radial basis function kernel. A nugget effect is included to account for the sampling variability of training samples due to the limited duration of simulation.

All the selected sea states are simulated with HOSM with the breaking model, using a JONSWAP wave spectrum and assuming infinite water depth. The numerical simulations with the improved 3D panel method

190 are performed for 3 hours in full scale. The results are affected by sampling variability due to limited duration of the simulations. Thus, the local peak values of ship bending moments are fitted with the twoparameter Weibull distribution using the method of moments. Finally, the 3-hour characteristic extreme value is estimated from the fitted Weibull distribution.

Nonlinear simulations are performed to calculate 3-hour characteristic extreme value of ship bending moment, capturing the asymmetry of ship hogging and sagging response. Thus, the emulator is established for ship hogging and sagging separately. The applicability of Gaussian processes regression is verified by choosing some sea states to estimate 3-hour characteristic extreme value of ship sagging and hogging moment with the 3D panel method for training the emulator and some sea states chosen for testing the emulator predictions. These selected sea states are illustrated in Figure 8. 

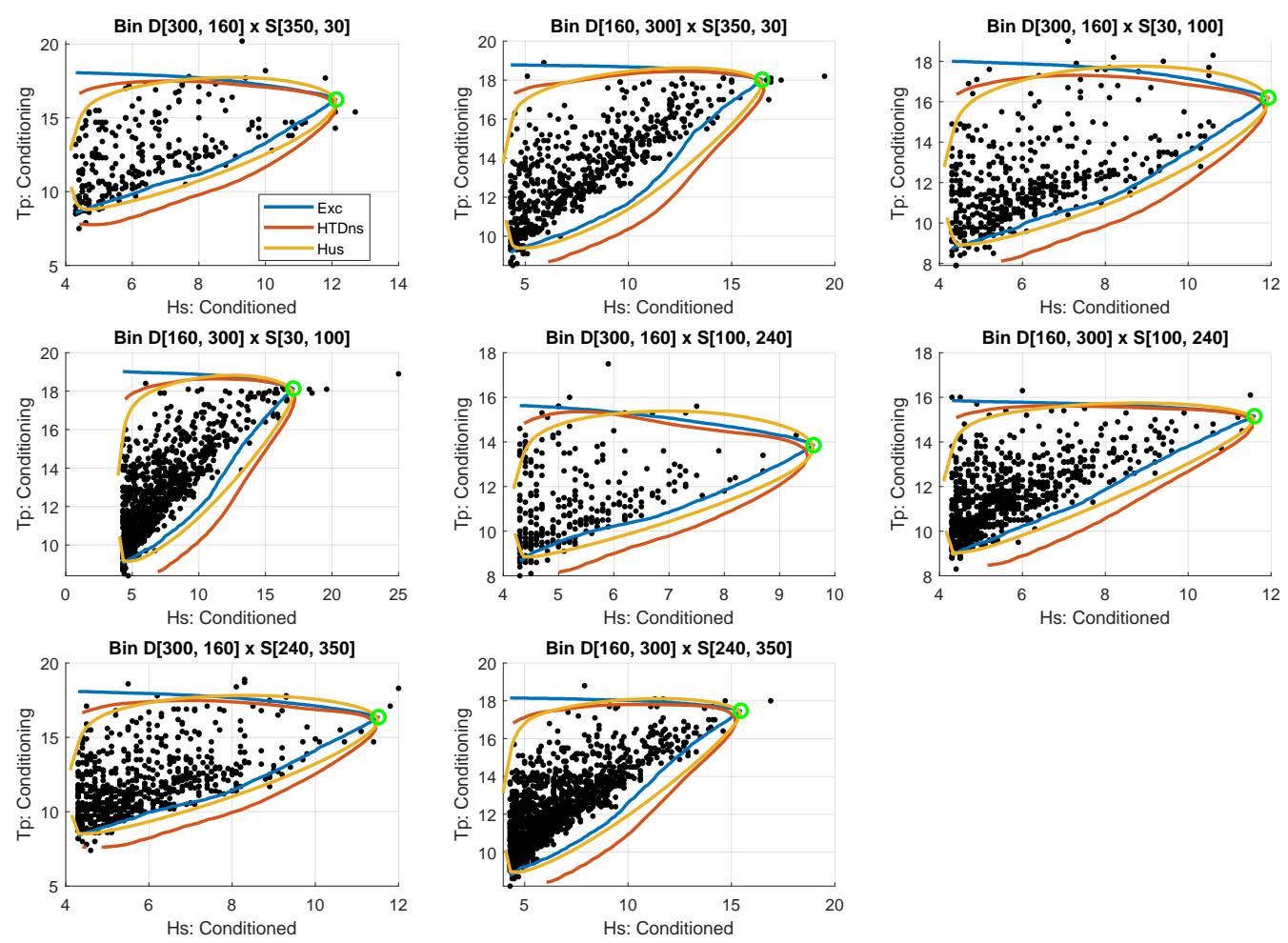

Figure 5: Environmental contours for selected directional-seasonal bins based on non-stationary analysis of the combined dataset

The fitted emulators for 3-hour extreme hogging and sagging are shown in Figure 9 . This shows the predicted responses (mean values) for different variations of $H_{S}$ and $T_{P}$, including $95 \%$ uncertainty bands. The training data are also indicated in the plots. These plots indicate that the Gaussian processes models capture the response surface quite well based on the training data.

Figure 10 compares emulator predictions with numerical results for hogging and sagging, respectively, in the test data. The calculated bending moments (true) from the improved 3D panel method is compared to predictions from the Gaussian processes model (pred). This illustrates that the emulator performs well and can predict responses for sea states not included in the training data. The differences between the predicted and the calculated responses are larger in steep sea states compared to less steep sea states. This is presumably due to the fact that the effect of sampling variability is larger in steeper wave conditions.

There are some small waves with $T_{P}$ less than $6 \mathrm{~s}$ in the wave data (see Figure 1). The bending moments will be very small for such conditions and will not be relevant for the 25-year extreme value. Moreover, linear simulations can give reasonable results for most of these wave conditions. In order to save computation cost, nonlinear simulations have not been carried out in this area and the emulator is therefore not extended to this area. However, linear calculations are utilized for these sea states in the response-based analysis.

\section{Extreme response estimation}

Having established a reasonable response emulator for the hogging and sagging bending moments, one may apply this to the complete time-series of environmental conditions and obtain equally long time-series of the structural response. Hence, 3 separate 30-year time series of hogging and sagging responses are obtained and extreme value analysis can be done on each of them in order to estimate the 25-year response from the time series. In addition, the response emulator can be used for all points along the various environmental contours in order to estimate the contour-based 25-year responses. 

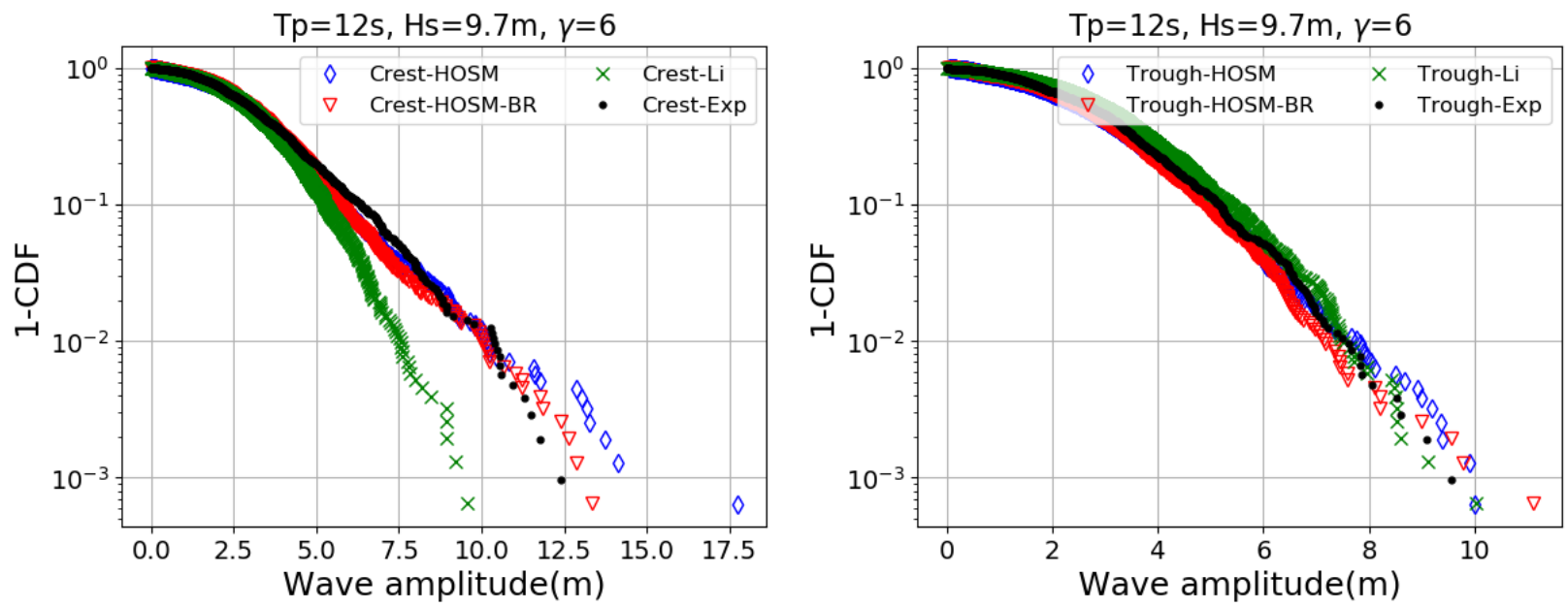

Figure 6: Comparison of simulated wave data and model tests for a selected irregular sea state with $T_{P}=12 \mathrm{~s}, H_{S}=9.7 \mathrm{~m}$ and $\gamma=6$; Wave crest (top) and wave trough (bottom)
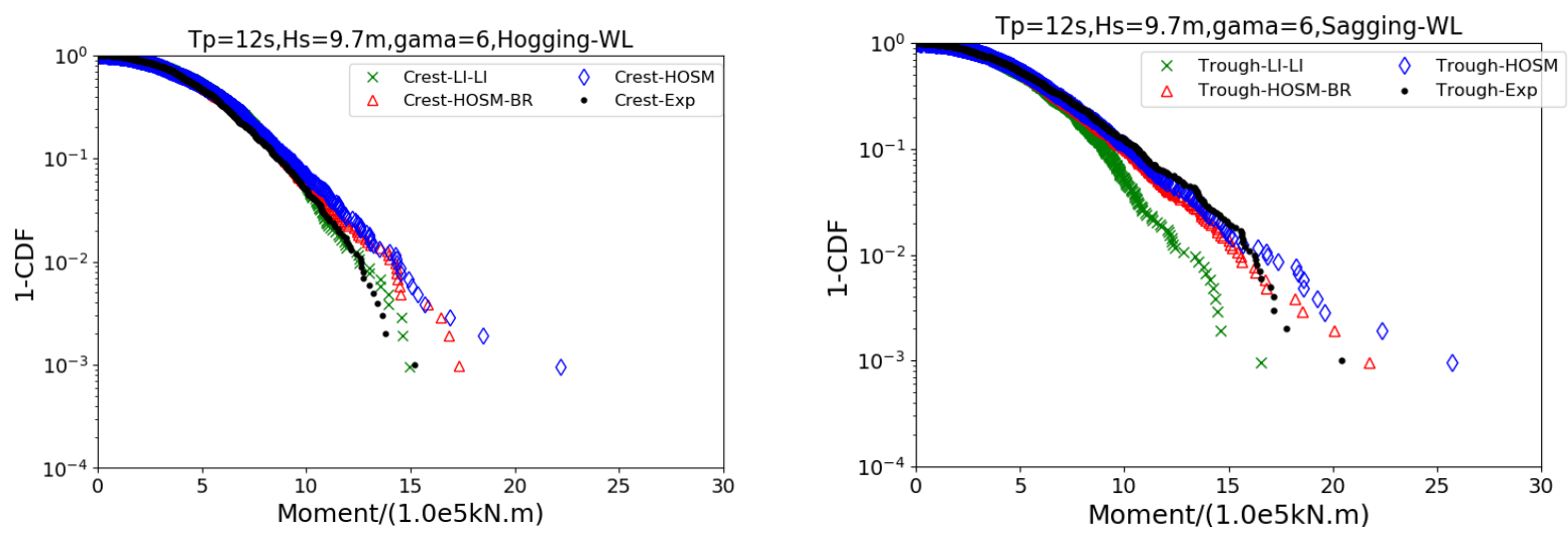

Figure 7: Comparison of mid-ship bending moment at waterline in irregular sea with $T_{P}=12 \mathrm{~s}, H_{S}=9.7 \mathrm{~m}$ and $\gamma=6$; Hoggind (top) and Sagging (bottom)

\subsection{Response based analysis}

The emulator provides long time-series of structural responses for hogging and sagging bending moment which may be used to estimate 25-year extreme responses. There are different ways of estimating extremes

225 from a long time-series and in this study the 25-year response is estimated in three ways. First, the empirical quantile can be found directly since the time series are longer than the target return period. For example, the 25 -year extreme can simply be estimated as the $1-1 /(25 \times 365.25 \times 8) \approx 0.9999863$-quantile from the empirical distribution. Secondly, a parametric distribution is fitted to all the response data, and the quantile according to the parametric model is calculated. It turns out that a 3-parameter Weibull distribution fits the data quite well, as illustrated by the QQ-plots in figure 11 for all the subsets of response data. Hence, quantiles from fitted 3-parameter Weibull distributions are used as alternative estimates of the 25-year response, where the fitting is done by minimizing the $2^{\text {nd }}$ order Anderson Darling statistic. Finally, the block maximum approach is used on annual maxima data from the response time-series to obtain yet other estimates of the 25-year response directly from the eumulated response time-series. Both the full GEV model and the reduced

235 Gumbel model are fitted to annual maxima by maximum likelihood to provide yet other point estimates of the 25-year responses. It is acknowledged that other methods exist for extreme value analysis, and that there are considerable uncertainties associated with this. However, the uncertainties will decrease as the amount of 


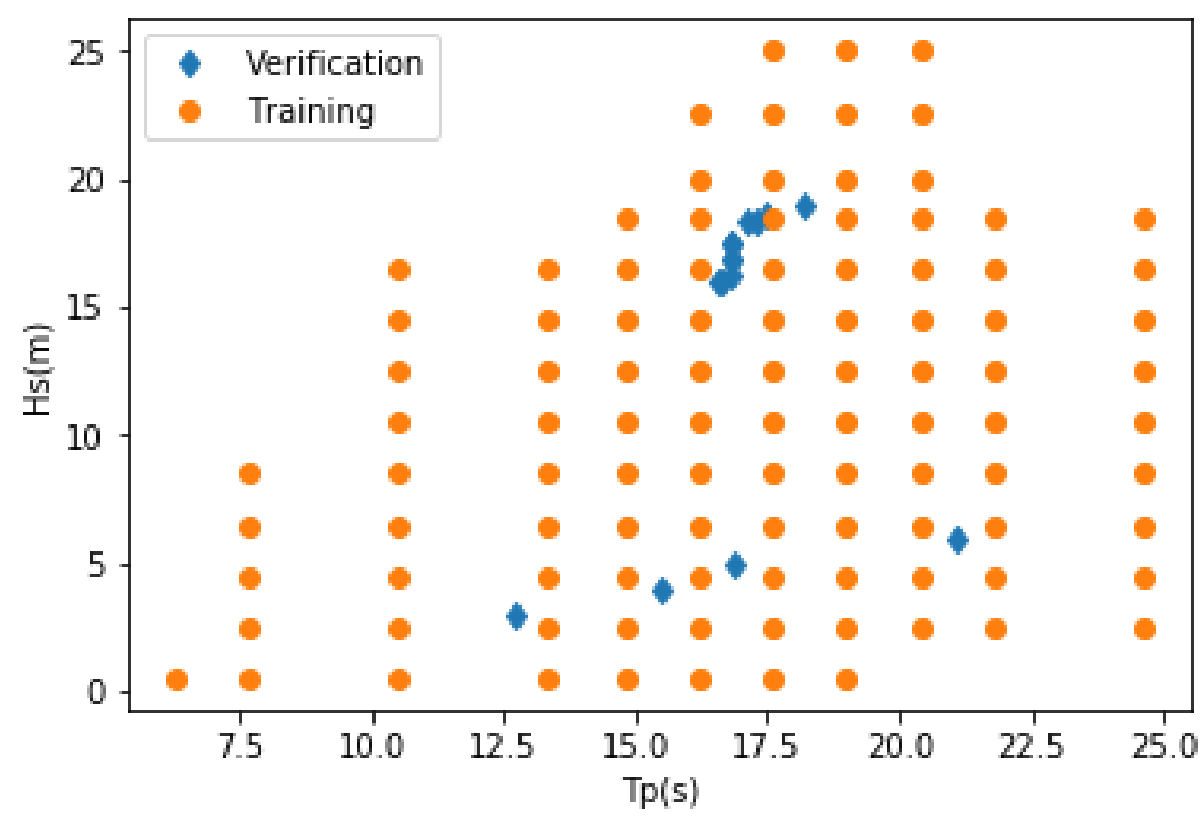

Figure 8: Sea states selected for training and testing the response emulator

Table 3: Estimated 25-year responses from response-based extreme value analysis (in $100 \mathrm{MNm}$ ).

\begin{tabular}{lcccc}
\hline Dataset & Empirical & Weibull & GEV & Gumbel \\
\hline \multicolumn{5}{c}{ Hogging } \\
r2 & 22.382 & 22.168 & 21.498 & 20.534 \\
r9 & 20.538 & 22.874 & 19.495 & 19.931 \\
r12 & 19.227 & 22.234 & 19.320 & 19.892 \\
Combined data & 20.895 & 22.364 & 20.214 & 20.215 \\
r2 & \multicolumn{5}{c}{ Sagging } \\
r9 & 27.519 & 27.049 & 26.191 & 24.324 \\
r12 & 24.663 & 28.092 & 23.122 & 23.538 \\
Combined data & 22.698 & 27.292 & 22.850 & 23.342 \\
& 25.347 & 27.371 & 24.124 & 23.875 \\
\hline
\end{tabular}

data increases, and estimates will also be extracted from the combined dataset corresponding to 90 years of data. This study only considers point estimates of the extreme response, but confidence bounds could easily 240 be found from bootstrapping.

The point estimates for 25 year hogging and sagging bending moments obtained from the response-based analysis are presented in Table 3. From these results, it is observed that there are considerable uncertainties due to sampling variability (different datasets) and different methods for extreme value extraction. Relative differences of up to $14 \%$ and $17 \%$ are seen for hogging and sagging responses, respectively, for the different datasets, where the largest differences are for the empirical estimates. Comparing the various methods for the individual datasets, the relative differences are up to almost $15 \%$ and $18 \%$ for hogging and sagging, respectively, and the largest differences are found for the r9 dataset. Surprisingly, the relative differences across methods are somewhat larger for the combined dataset compared to the r2 dataset.

One may also check QQ-plots for the GEV and Gumbel fits to the annual maximum data, and this is 250 shown in Figures 12 13. It can be observed that the full GEV model appear to fit better than the reduced Gumbel model, and indeed, Wald tests indicate that the Gumbel model (with $\xi=0$ ) would be rejected in all cases except for the hogging response for the combined dataset. 
Table 4: Estimated 25-year responses from contour-based analyses with initial distribution approach (in $100 \mathrm{MNm}$ ).

\begin{tabular}{lcccc}
\hline & \multicolumn{2}{c}{ Direct sampling contours } & \multicolumn{2}{c}{ IFORM contours } \\
& Hogging & Sagging & Hogging & Sagging \\
\hline r2 & 20.643 & 24.992 & 20.549 & 24.786 \\
r9 & 19.834 & 23.728 & 19.697 & 23.468 \\
r12 & 19.145 & 22.652 & 18.898 & 22.248 \\
Combined data & 20.365 & 24.578 & 20.253 & 24.329 \\
\hline
\end{tabular}

\subsection{Extreme response analysis from environmental contour method with initial distribution approach}

By applying the environmental contour method, one may obtain estimates of the 25-year responses from only a few short-term analyses by running response calculations for sea state conditions described by the environmental contour only. In this study, environmental contours have been calculated as described above, and the response emulator has been utilized to get the associated responses. The 25-year response is then simply taken as the maximum response along the contours. With the calculated environmental contours shown in Figure 2, the contour-based estimates of the 25-year responses in Table 4 are obtained.

It is observed that for all the datasets, the estimates obtained by the contour-method are comparable to the estimates from the response-based analysis. Indeed, except for the responses with the r12 dataset, the contour-based estimate lies within the range of values obtained by the different methods for response-based extreme value analysis. The relative differences (absolute value) between the various response-based estimates and the contour-based estimates range between $0.5 \%$ and $16 \%$ for the hogging response and between $0.9 \%$ and $20 \%$ for the sagging responses with the direct sampling contours and between $0.1 \%$ and $17 \%$ for the hogging response and between $0.8 \%$ and $22 \%$ for the sagging response with IFORM-based contours. The largest differences are in most cases for the estimates based on the Weibull fit to the response time-series, which also tend to be large compared to the other response-based estimates. It is also interesting to observe that the differences between the methods tend to be smaller for the combined dataset, indicating the importance of

270 sampling variability in extreme response estimation. Hence, environmental contours have been demonstrated to give reasonable, approximate return values of extreme bending moment in this case study.

\subsection{Extreme response analysis from environmental contour method with a conditional extremes model}

The conditional extremes model [34, 42] was also applied to the wave climate data as outlined above, and different contour methods have been applied to the results from this model. The resulting estimates of the 25-year extreme responses are presented in Table 5.

A non-stationary analysis was also performed on the combined dataset, where covariate effects due to varying direction (wind direction) and season were included in the model [43]. This results in environmental contours for different seasonal-directional bins, which could again be combined to yield omni-seasonaldirectional contours with the different contour methods (see Figures 5 and 4 ). The 25-year extreme responses corresponding to the omni-directional-seasonal contours are included in Table 5

It is found that the various contour methods applied together with the conditional extremes model compare reasonable well. However, the joint exceedance contours are found to give generally lower extreme responses than the other contour methods. Comparing the results from an initial distribution approach and the conditional extremes approach, it is also found that the results are comparable, and that the variability in the results are still dominated by the sampling variability. Indeed, all contour methods agree that dataset r2 yields higher extreme response estimates. This is also in agreement with the response-based estimates, apart from the ones based on a Weibull fit to all response data. Thus, also in this case, contour-based methods have been demonstrated to give reasonable results. An interesting observation is also that the stationary analysis seems to consistently give lower estimates of the 25-year extreme response compared to 290 the stationary analysis.

\section{Discussion}

\subsection{Conditions giving rise to extreme response}

Having estimated the 25-year extreme hogging and sagging bending moment using both response-based and contour-based methods, one may also look at which environmental conditions gave rise to the extreme 
Table 5: Estimated 25-year responses from contour-based analyses with conditional extremes model (in $100 \mathrm{MNm}$ ).

\begin{tabular}{lcccccc}
\hline & \multicolumn{2}{c}{ Direct sampling contours } & \multicolumn{2}{c}{ Joint exceedance contours } & \multicolumn{2}{c}{ Isodensity contours } \\
& Hogging & Sagging & Hogging & Sagging & Hogging & Sagging \\
\hline r2 & 21.53 & 26.42 & 21.23 & 25.61 & 21.65 & 26.57 \\
r9 & 19.62 & 23.41 & 18.71 & 21.80 & 19.59 & 23.33 \\
r12 & 19.81 & 23.71 & 19.27 & 22.57 & 19.93 & 23.80 \\
Combined data & 20.55 & 24.88 & 19.89 & 23.55 & 20.44 & 24.66 \\
Combined data; nonstationary analysis & 20.03 & 24.05 & 18.99 & 22.23 & 19.76 & 23.60 \\
\hline
\end{tabular}

Table 6: Conditions giving rise to the 25-year responses from contour-based and response-based analyses; $\left(H_{S}, T_{P}\right)$.

\begin{tabular}{|c|c|c|c|c|}
\hline & $\mathrm{r} 2$ & r9 & r12 & all \\
\hline Response-based & $(21.1,18.5)-(21.6,18.3)$ & $\begin{array}{c}\text { Hogging } \\
(18.9,18.2)-(19.4,18.2)\end{array}$ & $(16.0-16.6)-(17.7,18.0)$ & $(18.3,17.1)-(19.6,18.1)$ \\
\hline Initial distribution approach & & & & \\
\hline Direct sampling contours & $(18.54,17.51)$ & $(16.86,16.79)$ & $(16.22,16.83)$ & $(17.45,16.83)$ \\
\hline IFORM contours & $(18.80,17.80)$ & $(17.65,17.59)$ & $(16.46,17.25)$ & $(18.35,17.69)$ \\
\hline Conditional extremes appro & ach: & & & \\
\hline Direct sampling contours & $(19.54,17.52)$ & $(15.95,16.12)$ & $\begin{array}{l}(16.69,16.65) \\
\quad \text { Non-stationary: }\end{array}$ & $\begin{array}{l}(17.48,16.68) \\
(16.39,16.14)\end{array}$ \\
\hline Joint exceedance contours & $(20.63,18.65)$ & $(17.46,18.24)$ & $\begin{array}{l}(18.44,18.56) \\
\quad \text { Non-stationary: }\end{array}$ & $\begin{array}{l}(19.08,18.55) \\
(17.99,18.43)\end{array}$ \\
\hline Isodensity contours & $(19.88,17.69)$ & $(16.87,17.02)$ & $\begin{array}{l}(18.00,17.68) \\
\quad \text { Non-stationary: }\end{array}$ & $\begin{array}{l}(18.35,17.52) \\
(17.13,17.10)\end{array}$ \\
\hline $\begin{array}{l}\text { Response-based } \\
\text { Initial distribution approach }\end{array}$ & $(21.1,18.5)-(21.6,18.3)$ & $\begin{array}{c}\text { Sagging } \\
(18.9,18.2)-(19.4,18.2)\end{array}$ & $(17.7-18.0)-(16.0,16.6)$ & $(19.6,18.1)-(18.3,17.1)$ \\
\hline Direct sampling contours & $(18.29,17.30)$ & $(16.86,16.79)$ & $(15.98,16.62)$ & $(17.45,16.83)$ \\
\hline $\begin{array}{l}\text { IFORM contours } \\
\text { Conditional extremes appro }\end{array}$ & ach: $\quad(18.44,17.52)$ & $(17.19,17.22)$ & $(16.16,17.00)$ & $(18.01,17.42)$ \\
\hline Direct sampling contours & $(19.19,17.25)$ & $(15.95,16.12)$ & $\begin{array}{l}(16.48,16.45) \\
\quad \text { Non-stationary: }\end{array}$ & $\begin{array}{l}(17.48,16.68) \\
(16.39,16.14)\end{array}$ \\
\hline Joint exceedance contours & $(20.63,18.65)$ & $(17.33,18.14)$ & $(18.44,18.56)$ & $(19.08,18.55)$ \\
\hline Isodensity contours & $(19.88,17.69)$ & $(16.86,17.01)$ & $\begin{array}{l}\text { Non-stationary: } \\
(17.87,17.58) \\
\text { Non-stationary: }\end{array}$ & $\begin{array}{l}(17.71,18.21) \\
(17.85,17.13) \\
(17.13,17.10)\end{array}$ \\
\hline
\end{tabular}

295 6 For the response-based analysis, the associated sea-state conditi are based on the empirical quantile estimates and the conditions corresponding to the largest response lower than the quantile and the smallest response greater than the empirical 25-year response in the dataset are reported in the table.

It is observed that the response-based approach tends to identify more severe sea state conditions associated with the 25-year response compare to the contour-based methods. This may be due to the fact that such events occur in the tail of the probability distributions where there are very few observations. However, the environmental contour method construct a contour that also includes un-observed combinations of the environmental variables, whereas the response-based approach calculates the responses only for actually

305 observed sea state parameters. Hence, some combinations of environmental variables lying on the contour may not have been actually observed, and one may need to extend further out in the variable space to find the extreme responses. Notwithstanding, from Tables 35 it is seen that the empirical response-based return value estimates are slightly higher than the contour-based estimates, and this correspond to more severe sea state conditions, as seen in Table 6. This is illustrated in the sagging case in Figure 14, where the associated sea state conditions for the estimated extreme sagging response is illustrated (contour-results are shown for the initial distribution approach only). As can be seen, the contour-based estimates lie exactly on the environmental contours, whereas the sea states corresponding to the response-based estimates all lie outside the contours. The largest difference is observed for $\mathrm{r} 2$ scenario, whereas for $\mathrm{r} 12$, the lower response-based 
Table 7: Comparing response emulator with numerical model for selected sea states.

\begin{tabular}{lrrc}
$\left(H_{S}, T_{P}\right)$ & Numerical model & Emulator & Relative difference (\%) \\
\hline & \multicolumn{3}{c}{ Hogging } \\
$(18.534,17.500)$ & 20.68 & 20.643 & 0.18 \\
$(16.789,16.858)$ & 19.61 & 19.834 & -1.14 \\
$(16.205,16.816)$ & 18.82 & 19.145 & -1.73 \\
$(17.452,16.830)$ & 20.46 & 20.365 & 0.46 \\
& \multicolumn{3}{c}{ Sagging } \\
$(18.288,24.992)$ & 25.07 & 24.992 & 0.31 \\
$(16.858,16.789)$ & 23.76 & 23.728 & 0.13 \\
$(15.983,16.616)$ & 22.67 & 22.652 & 0.079 \\
$(17.452,16.830)$ & 24.41 & 24.578 & -0.69 \\
\hline
\end{tabular}

estimate coincides with the contour-based estimated conditions from the direct sampling contours. It may

315 seem strange that the 25-year response can be generated by a sea state with a much higher return period than 25 years, and this is probably an effect of the bivariate distribution function fitted to the wave data and used for constructing the environmental contours.

\subsection{Emulator response vs. numerical calculations for identified extreme conditions}

Both the response-based and the contour based extreme responses were estimated by the response emulator. In order to investigate how good an approximator this emulator is to the extreme responses, the responses corresponding to sea states along the environmental contours that give rise to the extreme responses are also calculated by the hydrodynamical model and compared to the results from the emulator. The calculated responses from the emulator and the hydrodynamic model for these selected $\left(H_{S}, T_{P}\right)$-pairs are shown in Table 7

325 It is observed that the emulator is a good approximator for the output from the hydrodynamical model, with relative differences less than $2 \%$ in all cases. This is negligible compared to the relative differences between the different results from various extreme value analysis methods on the emulated responses. Hence, the extra uncertainty due to the use of a response emulator is not significant in this case.

\subsection{Modelling sea states vs. storm peaks}

There is some debate within the user community regarding the relative merits of using observations of the environmental variables for serially-dependent sea states, compared with near-independent storm peak characteristics. Given that the rates of occurrence of events are taken into account, both can provide useful estimates of joint models for the environment and hence environmental contours. The advantage of using sea state data is that sample size is large, potentially allowing a more detailed description of the joint distribution

335 of environmental parameters to be estimated. However, because sea state data is serially-correlated, naive estimates of uncertainties for model parameters and inferences under the model will be too small, but can be corrected (for example, using sandwich estimators or bootstrap resampling).

\section{Summary and conclusions}

This paper explores response-based methods for extreme hogging and sagging response analysis of an LNG tanker, utilizing a response emulator based on Gaussian processes regression and compares with contour-based methods. The use of environmental contours for approximate analyses in structural reliability of marine structures is a well-recognized practice in the industry, and also response-based methods based on emulators have recently gain much attention. Hence, such a comparison is relevant to the industry.

The comparison of response-based and contour-based methods reveals that the approaches give comparable results and the differences are in the same order of magnitude as the difference between different extreme value analysis techniques applied to the response time-series with the response-based approach. Hence, both methods can be assumed to give reasonable approximations to the extreme response in this case. Also, by comparing the statistical response emulator to results from full hydrodynamical calculations for selected extreme sea states, it is found that the emulator is a good approximator to the numerical model. 
350 Notwithstanding, the uncertainties for extreme response estimation remains large, and in particular, the extrapolation of response data to extreme responses yields large uncertainties - even with quite long time-series corresponding to 30 and 90 years of data.

The sea states giving rise to the 25-year extreme responses have also bee investigated, and it is observed that the estimates from the response-based analysis correspond to sea-states well outside the 25-year environmental contours. This could be construed to mean that the 25-year response could be generated by sea-states with longer return period than 25 years. This may seem counter-intuitive, but could possibly be explained by the small amount of data in the tails of the distribution - even with quite long time-series and uncertainty due to how well parametric models fitted to the data describe tail behaviour. Such fitting is needed with both approaches, as they form the basis for environmental contours as well as response-based

360 extreme value analysis. Hence, in order to reduce uncertainties, more data would be recommended.

Different environmental contour methods have been compared, based on different modelling approaches for hte environmental variables, i.e. an all sea states approach and a storm peak approach. In the case study presented herein, all contour methods were found to give reasonable and comparable results. However, it is believed that in more complicated cases, for example with higher-dimensional problems, the differences

365 between the contour methods may be greater. Nevertheless, for the case study presented in this paper, it is difficult ti discriminate between the contour methods, and they are all found to perform reasonably well. Further investigations with more complex structural problems are recommended in order to investigate the relative merits of the different contour methods.

\section{Acknowledgement}

The work presented in this paper has been carried out within the research project ECSADES, with support from the Research Council of Norway (RCN) under the MARTEC II ERA-NET initiative; project no. $249261 / \mathrm{O} 80$.

\section{References}

[1] S. Haver, G. Kleiven, Environmental contour lines for design purposes - why and when?, in: Proc. 23rd International Conference on Offshore Mechanics and Arctic Engineering (OMAE 2004), American Society of Mechanical Engineers (ASME), 2004.

[2] S. Haver, On the joint distribution of heights and periods of sea waves, Ocean Engineering 14 (1987) $359-376$.

[3] S. Winterstein, T. Ude, C. Cornell, P. Bjerager, S. Haver, Environmental parameters for extreme response: Inverse FORM with omission factors, in: Proc. 6th International Conference on Structural Safety and Reliability, 1993.

[4] S. Haver, S. Winterstein, Environmental contour lines: A method for estimating long term extremes by a short term analysis, Transactions of the Society of Naval Architects and Marine Engineers 116 (2009) $116-127$.

[5] A. B. Huseby, E. Vanem, B. Natvig, A new approach to environmental contours for ocean engineering applications based on direct Monte Carlo simulations, Ocean Engineering 60 (2013) 124-135.

[6] A. B. Huseby, E. Vanem, B. Natvig, Alternative environmental contours for structural reliability analysis, Structural Safety 54 (2015) 32-45.

[7] E. Vanem, E. M. Bitner-Gregersen, Alternative environmental contours for marine structural design - a comparison study, Journal of Offshore Mechanics and Arctic Engineering 137 (2015) 051601:1-8.

[8] E. Vanem, A comparison study on the estimation of extreme structural response from different environmental contour methods, Marine Structures 56 (2017) 137-162.

[9] A. B. Huseby, E. Vanem, K. Eskeland, Evaluating properties of environmental contours, in: Proc. ESREL 2017, European Safety and Reliability Association(ESRA), 2017. 
[10] B. J. Leira, A comparison of stochastic process models for definition of design contours, Structural Safety 30 (2008) 493-505.

[11] A. F. Haselsteiner, J.-H. Ohlendorf, W. Wosniok, K.-D. Thoben, Deriving environmental contours from highest density regions, Coastal Engineering 123 (2017) 42-51.

[12] F. Silva-González, E. Heredia-Zavoni, R. Montes-Iturrizaga, Development of environmental contours using Nataf distribution model, Ocean Engineering 58 (2013) 27-34.

[13] K. R. Dahl, A. B. Huseby, Buffered environmental contours, in: Proc. ESREL 2018, European Safety and Reliability Association(ESRA), 2018.

[14] Q. Li, Z. Gao, T. Moan, Modified environmental contour method for predicting long-term extreme responses of bottom-fixed offshore wind turbines, Marine Structures 48 (2016) 15-32.

[15] R. Montes-Iturrizaga, E. Heredia-Zavoni, Environmental contours using copulas, Applied Ocean Research 52 (2015) 125-139.

[16] W. Chai, B. J. Leira, Environmental contours based on inverse SORM, Marine Structures 60 (2018) $34-51$.

[17] L. Manuel, P. T. Nguyen, J. Canning, R. G. Coe, A. C. Eckert-Gallup, N. Martin, Alternative approaches to develop environmental contours from metocean data, Journal of Ocean Engineering and Marine Energy 4 (2018) 293-310.

[18] E. Ross, O. C. Astrup, E. Bitner-Gregersen, N. Bunn, G. Feld, B. Gouldby, A. Huseby, Y. Liu, D. Randell, E. Vanem, P. Jonathan, On environmental contours for marine and coastal design, submitted.

[19] S. Wang, X. Wang, W. L. Woo, A comparison of response-based analysis and environmental contour methods for FPSO green water assessment, in: Proc. 37th International Conference on Ocean, Offshore and Arctic Engineering (OMAE 2018), American Society of Mechanical Engineers (ASME), 2018.

[20] E. Vanem, G. Bingjie, Comparison of the environmental contour method and response-based analysis using response emulator for estimating extreme ship responses, in: Proc. 38th International Conference on Ocean, Offshore and Arctic Engineering (OMAE 2019), American Society of Mechanical Engineers (ASME), 2019.

[21] A. B. Huseby, E. Vanem, B. Natvig, A new Monte Carlo method for environmental contour estimation, in: Proc. ESREL 2014, European Safety and Reliability Association(ESRA), 2014.

[22] E. Vanem, 3-dimensional environmental contours based on a direct sampling method for structural reliability analysis of ships and offshore structures, Ships and Offshore Structures 14 (2018) 74-85.

425 [23] P. Jonathan, K. Ewans, J. Flynn, On the estimation of ocean engineering design contours, Journal of Offshore Mechanics and Arctic Engineering 136 (2014) 041101:1-8.

[24] E. Vanem, Joint statistical models for significant wave height and wave period in a changing climate, Marine Structures 49 (2016) 180-205.

[25] E. Vanem, Uncertainties in extreme value modeling of wave data in a climate change perspective, Journal of Ocean Engineering and Marine Energy 1 (2015) 339-359.

[26] O. Gramstad, E. Vanem, E. Bitner-Gregersen, Uncertainty of environmental contours due to sampling variability, in: Proc. 37th International Conference on Ocean, Offshore and Arctic Engineering (OMAE 2018), American Society of Mechanical Engineers (ASME), 2018.

[27] R. Montes-Iturrizaga, E. Heredia-Zavoni, Assessment of uncertainty in environmental contours due to parametric uncertainty in models of the dependence structure between metocean variables, Applied Ocean Research 64 (2017) 86-104. 
[28] F. Silva-González, Vázques-Hernández, L. Sagrilo, R. Cuamatzi, The effect of some uncertainties associated to the environmental contour lines definition on the extreme response of an FPSO under hurricane conditions, Applied Ocean Research 53 (2015) 190-199.

[30] O. J. Aarnes, M. Reistad, Ø. Breivik, E. Bitner-Gregersen, L. I. Eide, O. Gramstad, A. K. Magnusson, B. Natvig, E. Vanem, Projected changes in significant wave height towards the end of the 21st century - Northeast Atlantic, Journal of Geophysical Research: Oceans 122 (2017) 3394-3403.

${ }_{445}$ [31] E. M. Bitner-Gregersen, E. Vanem, O. Gramstad, T. Hørte, O. j. Aarnes, M. Reistad, Ø. Breivik, A. K. Magnussen, B. Natvig, climate change and safe design of ship structures, Ocean Engineering 149 (2018) 226-237.

[32] E. M. Bitner-Gregersen, Joint met-ocean description for design and operation of marine structures, Applied Ocean Research 51 (2015) 279-292.

[33] K. Ewans, P. Jonathan, The effect of directionality on northern North Sea extreme wave design criteria, Journal of Offshore Mechanics and Arctic Engineering 130 (2008) 041604:1-8.

[34] J. E. Heffernan, J. A. Tawn, A conditional approach for multivariate extreme values, Journal of the Royal Statistical Society Series B 66 (2004) 497-546.

[35] E. Ross, S. Sam, D. Randell, G. Feld, P. Jonathan, Estimating sruge in extreme North Sea storms, Ocean Engineering 154 (2018) 430-444.

[36] D. Randell, K. Turnbull, K. Ewans, P. Jonathan, Bayesian inference for nonstationary marginal extremes, Environmetrics 27 (2016) 439-450.

[37] D. G. Dommermuth, D. K. P. Yue, A high-order spectral method for the study of nonlinear gravity waves, Journal of Fluid Mechanics 184 (1987) 267-288.

[38] B. J. West, K. A. Brueckner, R. S. Janda, D. M. Milder, R. L. Milton, Anew numerical method for surface hydrodynamics, Journal of Geophysical Research - Oceans 92 (1987) 11803-11824.

[39] B. Guo, E. M. Bitner-Gregersen, H. Sun, J. B. Helmers, Statistical analysis of ship response in extreme seas, Ocean Engineering 119 (2016) 154-164.

[40] W. Bateman, C. Swan, P. Taylor, On the calculation of the water particle kinematics arising in a directionally spread wavefield, Journal of Computational Physics 186 (2003) 70-92.

[41] B. Guo, O. Gramstad, E. Vanem, E. Bitner-Gregersen, Study on the effect of climate changes on ship responses based on nonlinear simulations, Journal of Offshore Mechanics and Arctic Engineering 141 (2019) 041605:1-13.

[42] K. Ewans, P. Jonathan, Evaluating environmental joint extremes for the offshore industry using the conditional extremes model, Journal of Marine Systems 130 (2014) 124-130.

[43] D. Randell, G. Feld, K. Ewans, P. Jonathan, Distributions of return values for the ocean wave characteristics in the South China Sea using directional-seasonal extreme value analysis, Environmetrics 26 (2015) 442-450. 

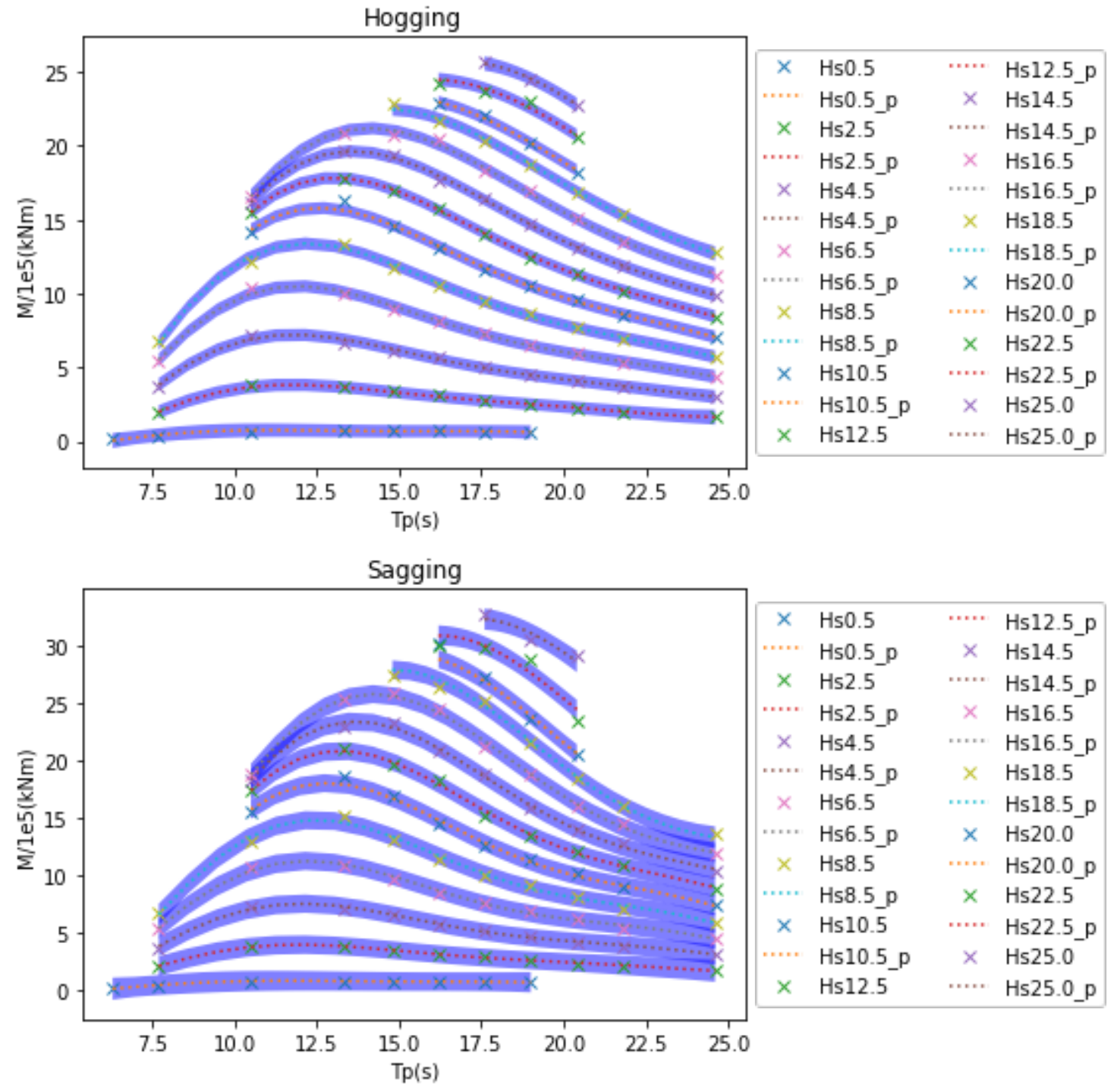

Figure 9: Response surfaces as predicted by the Gaussian processes emulators; hogging (top) and sagging (bottom). The crosses denote the training data 

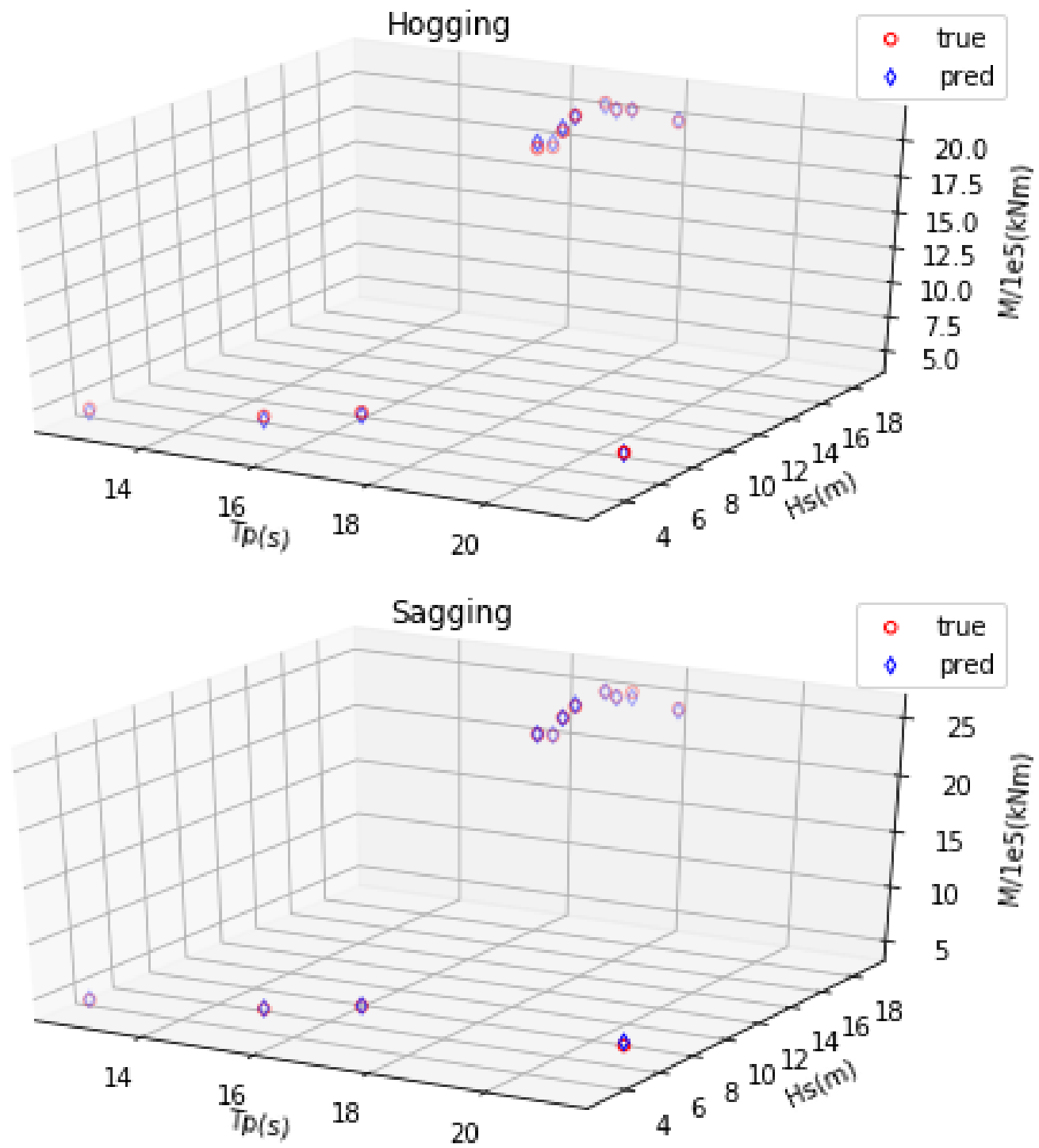

Figure 10: Comparing emulator predictions on the test data; hogging (top) and sagging (bottom). 

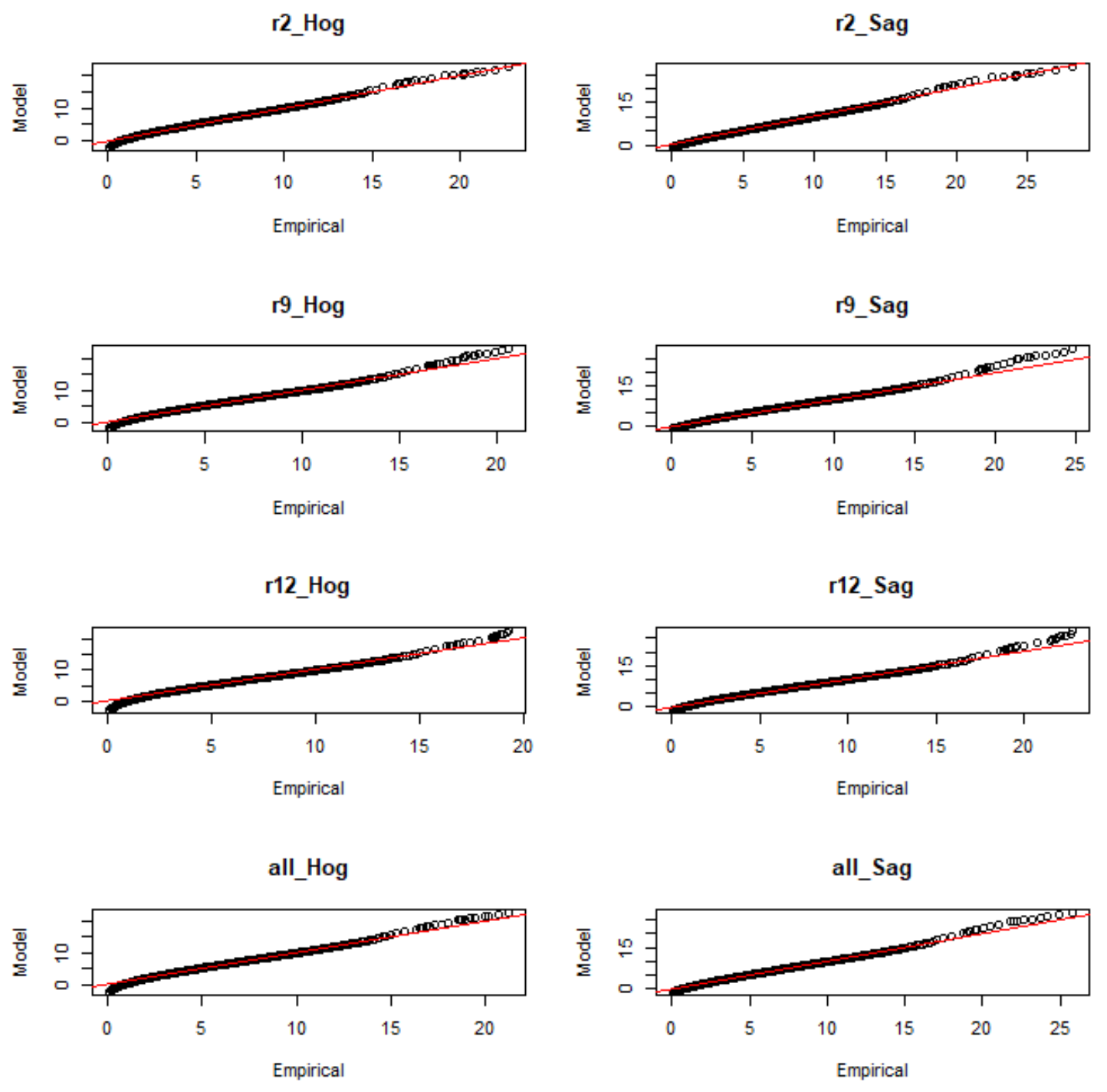

Figure 11: QQ-plots for the 3-parameter Weibull distribution for the different datasets; hogging (left) and sagging (right) 

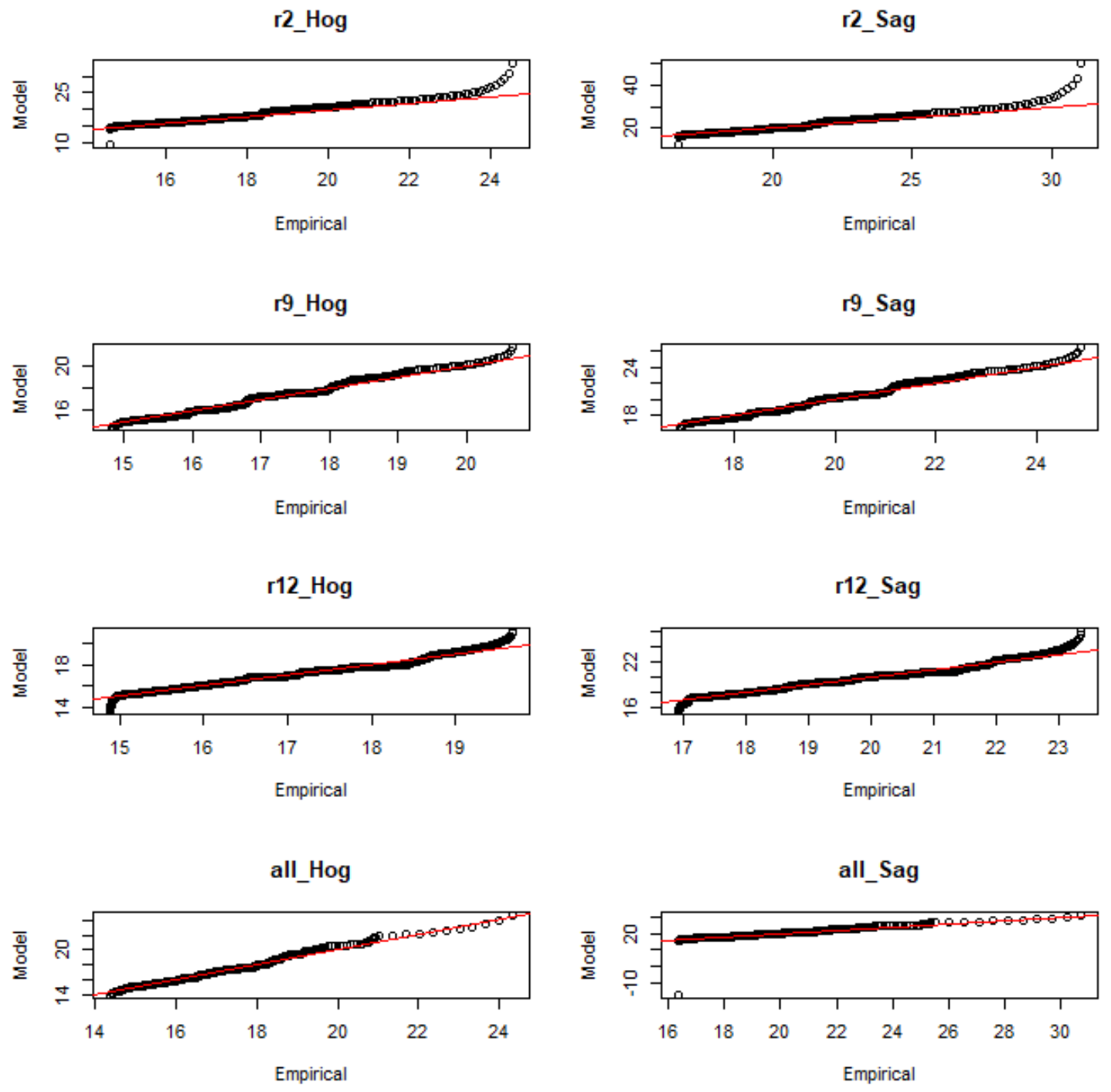

Figure 12: QQ-plots for the GEV model fitted to annual maxima; hogging (left) and sagging (right) 

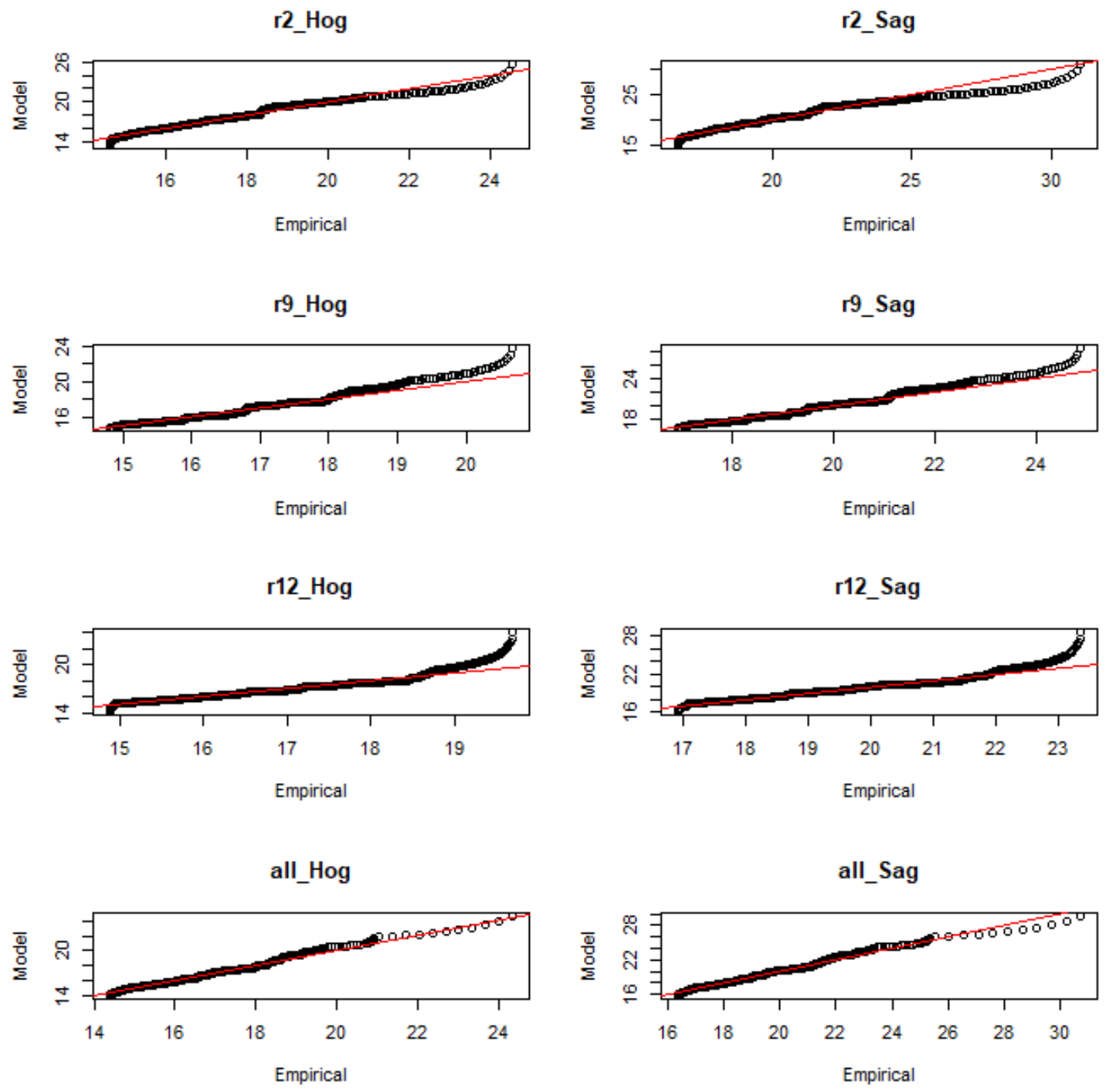

Figure 13: QQ-plots for the Gumbel model fitted to annual maxima; hogging (left) and sagging (right) 


\section{Environmental conditions 25-year sagging}

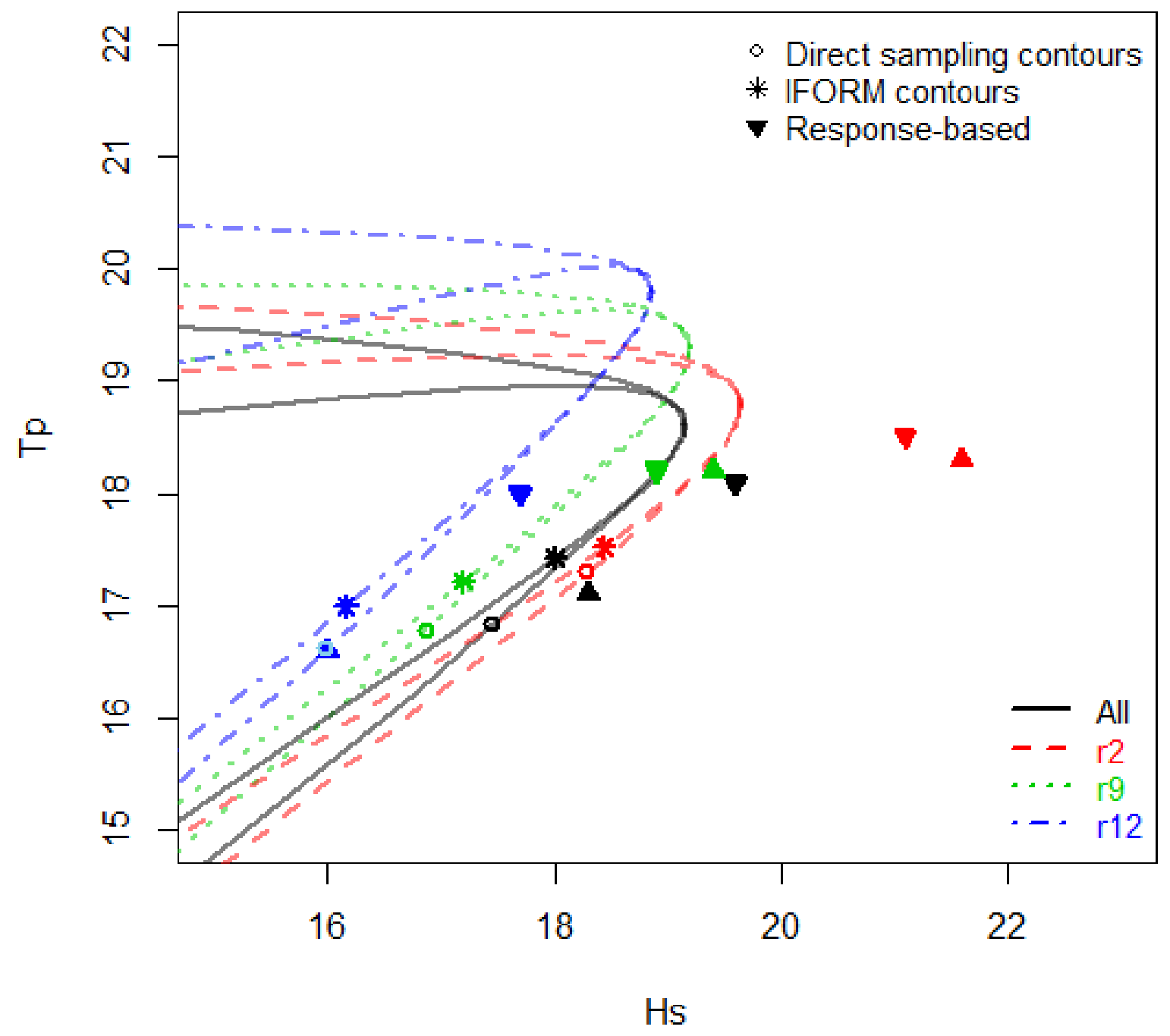

Figure 14: Sea states associated with extreme sagging responses 